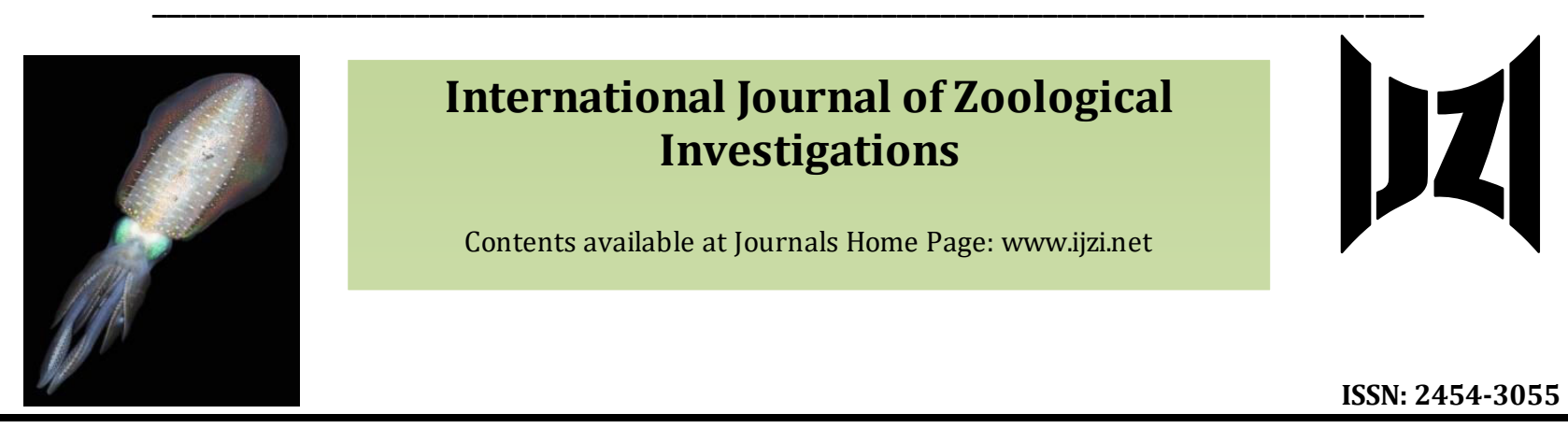

\title{
Assessment of Toxicity of Coragen, an Insecticide, on Aquatic Ecosystem Using Hydra as a Model
}

\author{
Borgave Seema Sachin \\ Post-graduate Department of Zoology, Sangamner Nagarpalika Arts, D. J. Malpani Commerce and B.N. Sarda Science College \\ (Autonomous), affiliated to Savitribai Phule Pune University, Sangamner 422605, Ahmednagar, Maharashtra, India
}

Received: $1^{\text {st }}$ March, 2021

Accepted: $2^{\text {nd }}$ April, 2021

Published online: $4^{\text {th }}$ April, 2021

https://doi.org/10.33745/ijzi.2021.v07i01.012

\begin{abstract}
Overuse of ins ecticides, without analyzing their harmful effects, can cause serious environmental issues. Coragen is a commonly used insecticide due to its broad range activity. In spite of enormous use, there are hardly any reports on the effects of coragen on non-target aquatic species and thus the environmental risk of coragen to aquatic ecosystem is underestimated. The present study was carried out to evaluate the potential risk of coragen on the water bodies and the life therein using hydra, a fresh water simple diploblastic invertebrate, as the model system. Coragen was found to reduce the life expectancy of hydra along with the reduced ability to regenerate at various tried doses. The present study thus suggests precocious use of coragen due to its harmful effects on aquatic animals at very low concentrations also. The continued overuse of coragen may disturb the aquatic ecosystems at a large scale in long run.
\end{abstract}

Keywords: Coragen, Hydra, Lethal concentration, Regeneration, Toxicity

Citation: Borgave Seema Sachin: Assessment of toxicity of coragen, an insecticide, on aquatic ecosystem using hydra as a model. Intern. J. Zool. Invest. 7 (1): 159-168, 2021.

https://doi.org/10.33745/ijzi.2021.v07i01.012

\section{Introduction}

Persistent, though slow, release of chemical pesticides and fertilizers may accumulate in the nearby water bodies, thus change its quality and may turn it inappropriate for the life present therein (Staley et al., 2015; Solis et al., 2018). Coragen, (chlorantraniliprole), is one of the most widely used insecticides due to its broad spectrum of activity. It is effective against immature and adult stages of many key pests like European corn borers, armyworm, black cutworm, Colorado potato beetle etc. Coragen protects more than 100 vegetable crops by controlling infestation of variety of insect pests, mainly Lepidopteran, 
probably by affecting nervous system and interrupting feeding of these pests immediately after coragen exposure. Coragen activates ryanodine receptors by the stimulation of calcium, causing paralysis and death of the sensitive species (Mishra et al., 2016). In spite of being a widely applied insecticide with potential long term effects on environment, there are hardly any reports describing toxicological aspects of coragen on non-target aquatic species.

Hydra, a freshwater cnidarian, has been explored as one of the most suitable model for studying the aquatic toxicology (Karntanut and Pascoe, 2002). It has an unparalleled ability to regenerate, allowing the assessment of teratogenic compounds and the impact of contaminants on stem cells as well. The most important feature of hydra is that they are pervasive in freshwater bodies and are comparatively easy to culture in laboratory. Thus, hydra has emerged as an aquatic environmental indicator and for small scale bioassays (Quinn et al., 2012; Riedl et al., 2018). In the context to enormous use of coragen in India, the present work was undertaken to investigate the possible illeffects, if any, of coragen on non-target aquatic species using hydra as a model system. The study evaluated effects of corgaen on whole hydra, regenerating hydra and estimated lethal concentration $50 \%$ of coragen for the test animal.

\section{Materials and Methods}

Culture of hydra: A clonal culture of Chlorohydra viridissima (green hydra) was maintained at room temperature in the laboratory in clean glass beakers filled with double filtered and autoclaved pond water.
Zooplanktons (Paramoecium, Daphnia, Cyclops etc.) were used for feeding hydra on alternate days. The leftover food and dead debris were cleaned $5 \mathrm{~h}$ post-feeding.

Preparation of treatment doses of coragen: Coragen was procured from a local shop and serially diluted $(1: 10,1: 100,1: 1000,1: 10000$ and 1:100000 dilution) using filtered sterile pond water to prepare the treatment solutions with various potencies ranging from $18400,1840,184,18.4$ and $1.84 \mathrm{ppm}$ of coragen, respectively.

Treatment of whole hydra with coragen: Fully grown healthy hydra were selected and randomly distributed in equal numbers into the beakers containing equal volume of filtered sterile pond water with or without known concentration of coragen. Hydra, kept in pond water alone served as controls and rest were used for treatment with various concentrations, ranging from 18400 to 1.84 ppm of coragen. The death rate, overall cellular morphology and contractibility of hydra from all the beakers was monitored over a period of $72 \mathrm{~h}$ post-treatment. Detailed cellular morphology was observed under Leica DM 1000 microscope (DST-FIST instrument) and the observations were recorded using Future WinJoe camera. The experiment was repeated for 5 times with 10 hydra in each beaker every time.

Treatment of regenerating hydra with coragen: Full-grown hydra were selected and starved for 2 days for the experiments. Hydra were cut transversely in the middle of the body column using tungsten needles. The two body column pieces generated were with either hypostome or basal disc. These pieces were separated on the basis of hypostome and basal disc, and randomly distributed into 
separate beakers for treatment with or without coragen. One beaker was kept as a control and the other was treated with 1:100000 dilution (1.84 ppm) of coragen. In this way, the experimental design had 4 beakers; one beaker with regenerating hypostome pieces without coragen, second beaker with regenerating hypostome pieces with coragen, third beaker with regenerating basal pieces without coragen and the last beaker with regenerating basal disc pieces with coragen. The regeneration in all the groups was monitored on the basis of morphological and functional criteria for next $72 \mathrm{~h}$ and the observations were recorded. The hydra were not fed during this period. All the experiments were repeated minimum 5 times with minimum 10 pieces in each control and treatment beakers every time.

\section{Results}

The effects of dilutions of coragen on whole hydra and regenerating hypostome or basal disc pieces were followed for $72 \mathrm{~h}$ posttreatment. Observations were recorded at the end of every $24 \mathrm{~h}$.

Effects of coragen on whole hydra: Hydra from the control group exhibited normal morphology and contractibility throughout the experiment. Lethal effects of coragen, at all the tried doses, were recorded as depicted in Table 1. Coragen (1.84 ppm) was found to kill approximately 50 per cent of the treated hydra by $72 \mathrm{~h}$. In this way, the $\mathrm{LC}_{50}$ of coragen on whole hydra for $72 \mathrm{~h}$ was estimated to be 1:100000 dilution i.e. 1.84 ppm (Table 1).

The overall morphology of the body column, tentacles, attachment and feeding pattern of hydra from the control group was normal throughout the experiment (Figs. 1 A, 2 A, 3 A). Coragen, at 18400 ppm concentration, completely disintegrated the hydra whereas hydra treated with 1840 ppm were immotile with partial loss of the tentacles and disintegrated body column cells within 24 h (Fig. 1 B). They were considered dead due to absence of reflex action. At the 184 and 18.4 ppm concentrations of coragen, the cells of the body column and tentacles appeared to loosen up by $24 \mathrm{~h}$ with impaired movement of tentacles (Figs. 1 C, D). The morphology of body column cells and tentacles was found to deteriorate as compared to respective controls when the treatment period was extended beyond $24 \mathrm{~h}$ for these concentrations (Figs. 2 A, C, D, 3 A, C, $D)$. The morphology of hydra in $1.84 \mathrm{ppm}$ coragen treated group was comparable to the respective controls for initial $48 \mathrm{~h}$ (Figs. 1; A, E, 2 A, E). By the end of $72 \mathrm{~h}$, hydra treated with 1.84 ppm concentration of coragen also showed indications of cellular disintegration with death of $50 \%$ of the treated hydra (Fig. $3 \mathrm{E})$. Coragen treatment led to deterioration of the contractibility and relaxation pattern of hydra as compared to the respective controls within $72 \mathrm{~h}$.

Effects of coragen on regenerating hydra: Hydra were cut transversely by taking a sharp cut along the body axis as depicted in Figure 4. The regeneration of basal disc in the pieces with hypostome, and hypostome in the pieces with basal disc was monitored every $24 \mathrm{~h}$. During regeneration, the blastema formation begins immediately within $24 \mathrm{~h}$ and the rearrangement of body column cells to reform the lost parts is achieved within $72 \mathrm{~h}$. Since $1.84 \mathrm{ppm}$ concentration of coragen was detected as $\mathrm{LC}_{50}$ for whole hydra (72 h), the same dose was used for treatment of regenerating body pieces of hydra. The overall regeneration was achieved with 
Table 1: Effects of coragen at various concentrations on whole hydra

\begin{tabular}{ccccccc}
\hline Treatment window & Control & $\mathbf{1 8 4 0 0} \mathbf{p p m}$ & $\mathbf{1 8 4 0} \mathbf{p p m}$ & $\begin{array}{c}\mathbf{1 8 4} \\
\mathbf{p p m}\end{array}$ & $\begin{array}{c}\mathbf{1 8 . 4} \\
\mathbf{p p m}\end{array}$ & $\mathbf{1 . 8 4} \mathbf{p p m}$ \\
\hline $\mathbf{2 4} \mathbf{h}$ & $100 \%$ & $0 \%$ & $0 \%$ & $11.11 \%$ & $50 \%$ & $88.88 \%$ \\
\hline $\mathbf{4 8} \mathbf{h}$ & $100 \%$ & $0 \%$ & $0 \%$ & $11.11 \%$ & $50 \%$ & $61.11 \%$ \\
\hline $\mathbf{7 2} \mathbf{h}$ & $100 \%$ & $0 \%$ & $0 \%$ & $5.55 \%$ & $33.33 \%$ & $\mathbf{5 0} \%$ \\
\hline
\end{tabular}

The percentage given here depicts the overall per cent of live hydra in each category of coragen concentration at the end of the treatment window. Note no live hydra at higher doses of coragen i.e. 18400 ppm and $1840 \mathrm{ppm}$ within $24 \mathrm{~h}$. LC 50 of coragen for hydra for $72 \mathrm{~h}$ was observed to be $1.84 \mathrm{ppm}$.

Table 2: Effects of coragen (1.84 ppm) on regeneration of hydra

\begin{tabular}{ccccccc}
\hline \multirow{2}{*}{ Treatment window } & \multicolumn{2}{c}{ After $\mathbf{2 4} \mathbf{~ h}$} & After $\mathbf{4 8} \mathbf{~ h}$ & After $\mathbf{7 2} \mathbf{~ h}$ \\
\cline { 2 - 6 } & Hypostome & Basal Disc & Hypostome & Basal Disc & Hypostome & Basal Disc \\
\hline Control & $100 \%$ & $100 \%$ & $97.61 \%$ & $100 \%$ & $97.61 \%$ & $100 \%$ \\
\hline Coragen & $95.23 \%$ & $95.23 \%$ & $80.95 \%$ & $85.71 \%$ & $76.19 \%$ & $66.15 \%$ \\
\hline
\end{tabular}

The percentage is calculated on the basis of regeneration shown in the hypostome and basal disc pieces of live hydra in each category. Note reduction in percentage of regeneration in hypostome pieces and basal disc pieces after coragen treatment as compared to the respective controls after $72 \mathrm{~h}$ regeneration.

formation of normal structures in the control groups whereas the regeneration of the hypostome and basal disc pieces, both were hampered due to coragen (Table 2).

The body column pieces with hypostome, started regeneration of the lost basal discs in the control group within $24 \mathrm{~h}$ (Fig. 5 A). Hypostome exhibited formation of functional basal discs by $72 \mathrm{~h}$ (Figs. 5 B, C). The hydra could attach to the glass surface with the regenerated basal discs. On the other hand, hypostome pieces treated with coragen showed signs of blastema formation and beginning of basal disc regeneration after $24 \mathrm{~h}$ (Fig. 5 D). However, later on the body cells showed disintegration to some extent (Figs. $5 \mathrm{E}, \mathrm{F})$. In the control group, the basal disc pieces started hypostome regeneration by the end of $24 \mathrm{~h}$ (Fig. $6 \mathrm{~A}$ ). The regenerated hypostome and tentacles exhibited normal morphology in control group (Figs. 6 B, C). On the contrary, coragen treated basal disc pieces showed regeneration of hypostome with miniature tentacles with disturbed morphology (Figs. 6 D, E). The regenerated hydra exhibited no or low movements in 


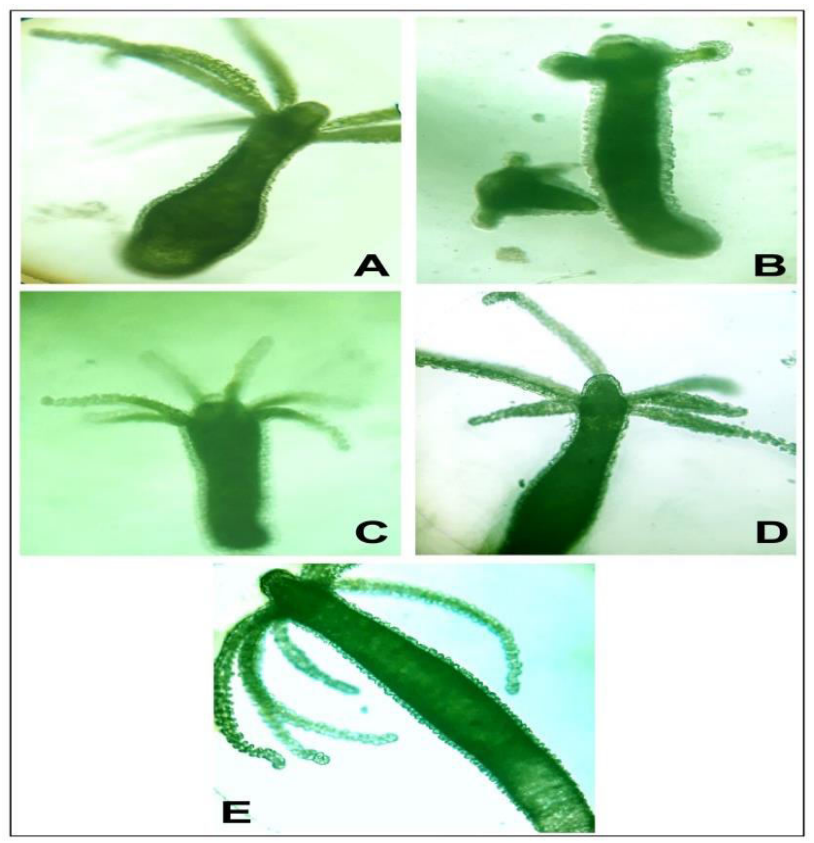

Figure 1: Effects of various concentrations of coragen on whole hydra (24 h): (A) Control hydra; note control hydra with normal morphology of body column and tentacles, (B-E) Coragen treated hydra - (B) 1840 ppm; observe disintegration of tentacles and distorted body column, (C) 184 dilution and (D) 18.4 ppm; note loosening of cells in tentacles, (E) 1.84 ppm; observe hydra morphology comparable with control as seen in A.

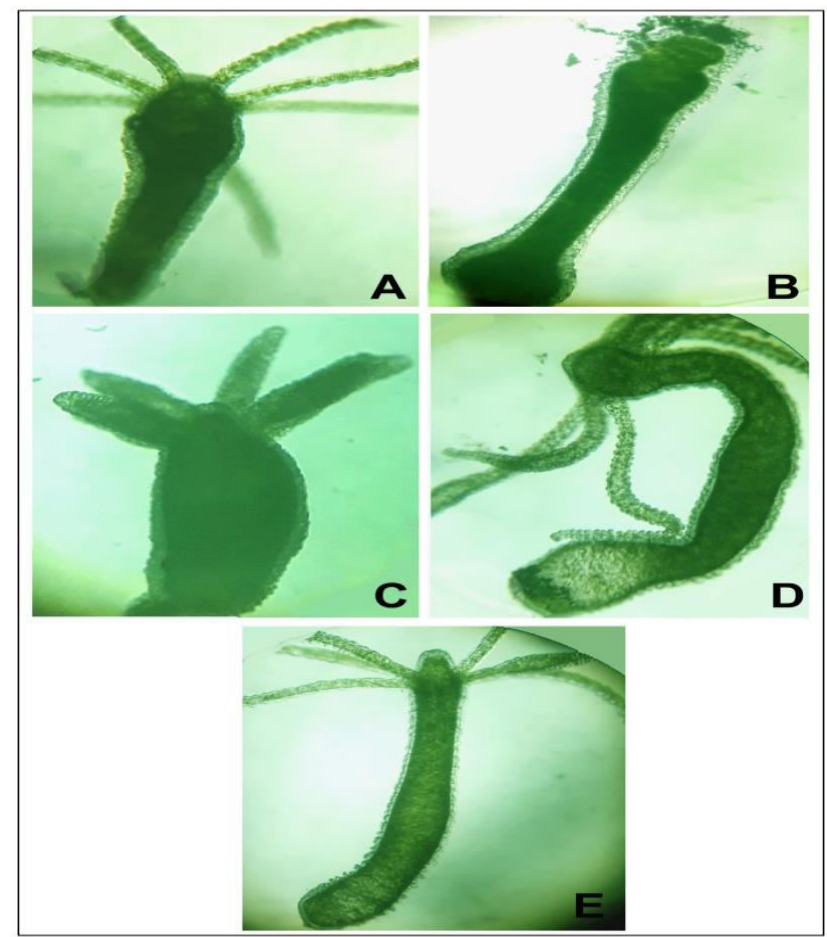

Figure 2: Effects of various concentrations of coragen on whole hydra (48 h): (A) Control hydra with normal cellular morphology, (B-E) Coragen treated hydra - (B) $1840 \mathrm{ppm}$; note complete loss of tentacles and immobile body column, (C) 184 ppm; observe abnormal tentacles and body column morphology, (D) 18.4 ppm; observe loosening of cells of tentacles and basal disc region, (E) 1.84 ppm; beginning of distortion in cell morphology in tentacles and body column cells was evident. 


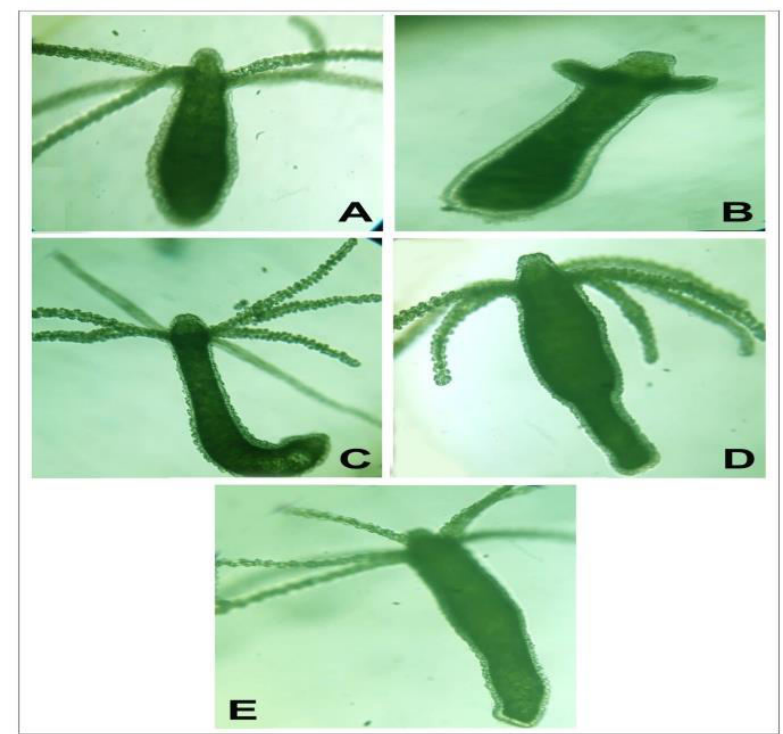

Figure 3: Effects of various concentrations of coragen on whole hydra (72 h): (A) Control hydra exhibiting normal cellular morphology all over body, (B-E) Coragen treated hydra - (B) 1840 ppm; though the hydra were dead within $24 \mathrm{~h}$ the body did not disintegrate completely by $72 \mathrm{~h}$ (C) $184 \mathrm{ppm}$ and (D) 18.4 ppm; note disturbed cellular morphology of body column and tentacles, (E) 1.84 ppm; observe advancement in loosening of cells as compared to $48 \mathrm{~h}$ time point.

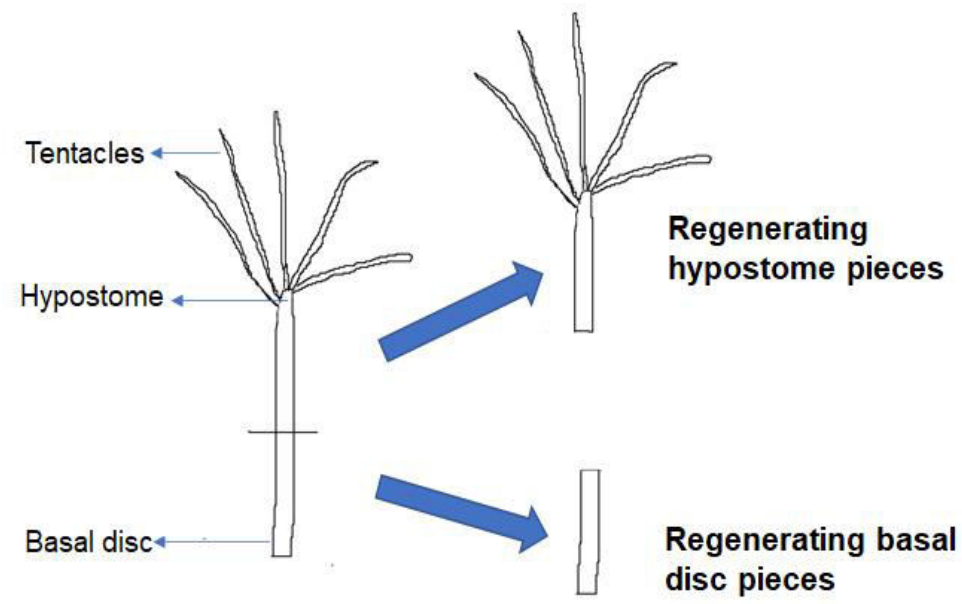

Whole hydra showing the position of transverse cut

Figure 4: Diagrammatic representation of cutting of whole hydra into 2 pieces that were used for regeneration experiments. Out of the two pieces generated this way, one was the regenerating hypostome piece with tentacles while the other piece was the regenerating basal disc. The regenerating hypostome piece would reform the lost basal disc while the regenerating basal disc would regenerate the lost hypostome region with tentacles in due course of time. 


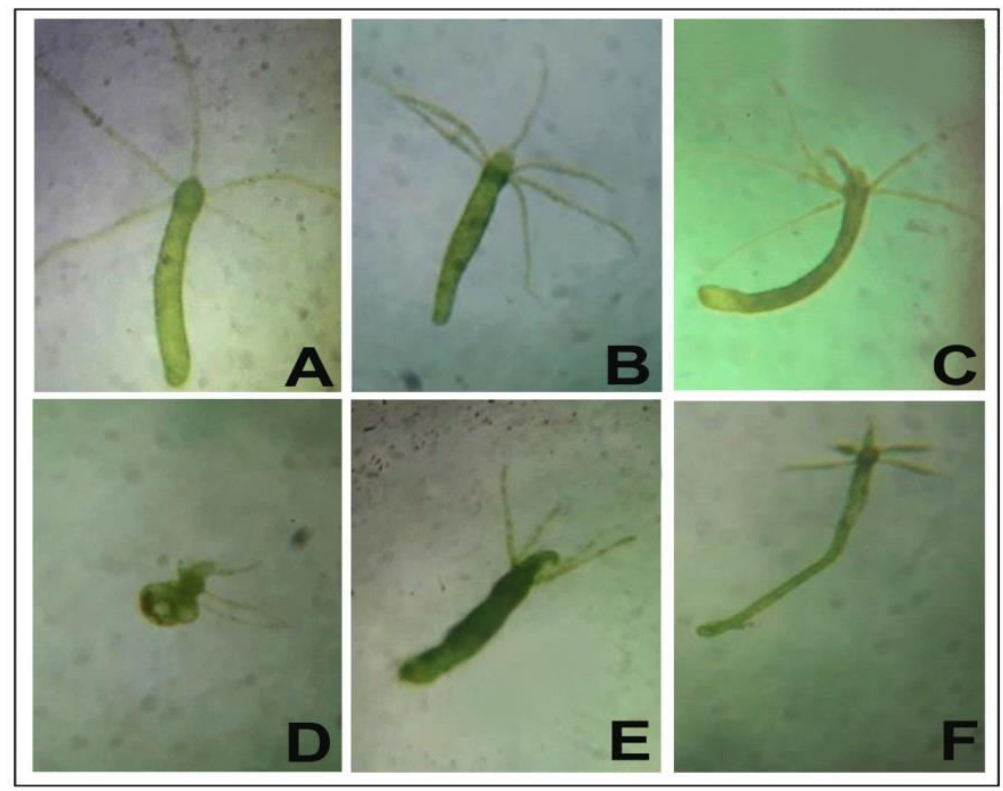

Figure 5: Effect of $1.84 \mathrm{ppm}$ of coragen on regenerating hypostome pieces: (A) control hypostome after $24 \mathrm{~h}$, (B) control hypostome after $48 \mathrm{~h}$, (C) control hypostome after $72 \mathrm{~h}$, (D) coragen treated hypostome after $24 \mathrm{~h}$, (E) coragen treated hypostome after $48 \mathrm{~h},(\mathrm{~F})$ coragen treated hypostome after $72 \mathrm{~h}$; note formation of functional basal disc in controls (C) by $72 \mathrm{~h}$ whereas the formation of basal disc was delayed in treated hydra. Also, the tentacles were damaged by $72 \mathrm{~h}$ in these cases (F).

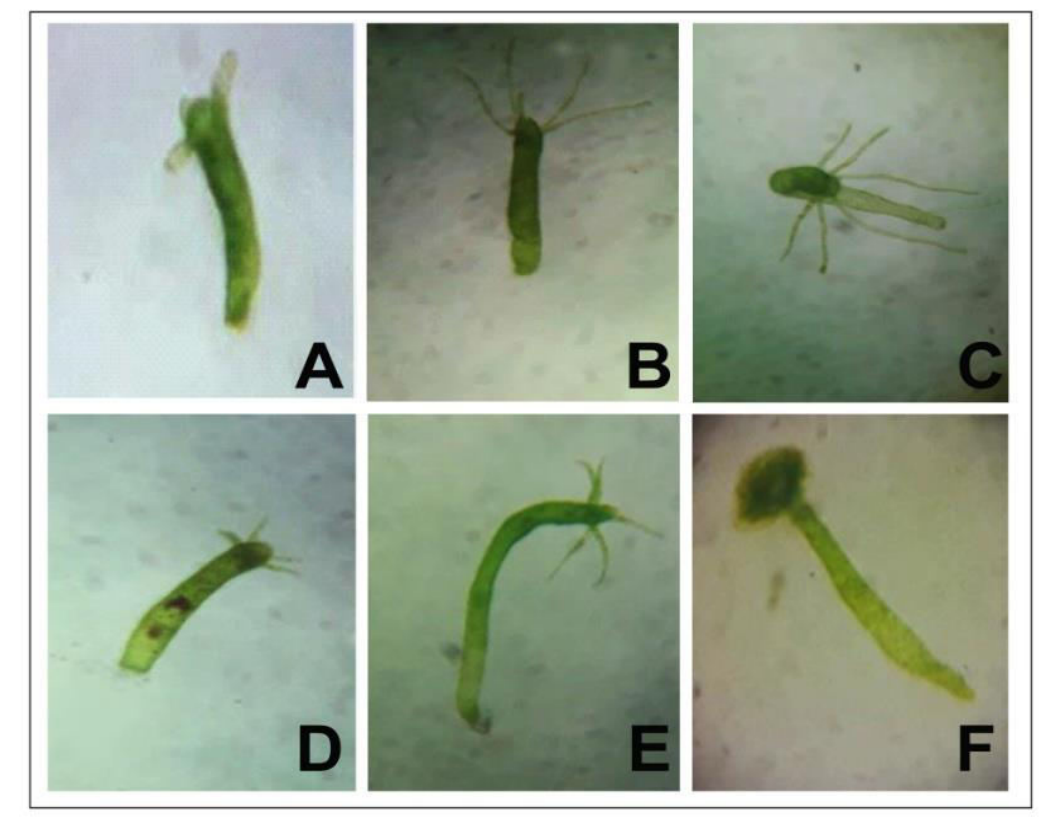

Figure 6: Effects of $1.84 \mathrm{ppm}$ of coragen on regenerating basal disc pieces: (A) control basal disc after $24 \mathrm{~h}$, (B) control basal disc after $48 \mathrm{~h}$, (C) control basal disc after $72 \mathrm{~h}$; observe the length and compact arrangement of cells in the regenerated tentacles, (D) coragen treated basal disc after $24 \mathrm{~h}$ (E) coragen treated basal disc after $48 \mathrm{~h}$; note miniature tentacles as compared to respective control (B), (F) coragen treated basal disc after $72 \mathrm{~h}$; complete disintegration of the regenerating tentacles was observed in about $30 \%$ of the piec es as shown here while in most of the cases the cells of the tentacles were not compactly arranged as compared to C. 
coragen treated groups as compared to the respective controls. Coragen, thus was found to interfere with the regeneration process at the tried dose in the present study.

\section{Discussion}

Hydra is the most widely used model animal for aquatic toxicity investigations as it acts as one of the crucial members of the food chain operating in stagnant fresh water bodies (Quinn et al., 2012; Blaise et al., 2018; McKinley et al., 2019). Most of chemicals used as agricultural aids entre into nearby water bodies as runoff water. This may pollute the water bodies making them unsuitable for the biotic components of the ecosystem. In this scenario, the present study was undertaken to address the toxic effects of the most commonly used insecticide, coragen on aquatic ecosystem using hydra as a model.

Ryanodine receptors, located in the sarcoplasmic reticulum, function in calcium induced calcium release (CICR) in excitable tissues like muscles (Bouchard et al., 2003). Activation of ryanodine receptors by coragen (active ingredient - chlorantraniliprole), resulting in paralysis and ultimately death of the susceptible species has been demonstrated (Dutta et al., 2014; Mishra et al., 2016). Coragen causes cardiac problems and other metabolic abnormalities related to magnesium and calcium also (Mishra et al., 2016). In the present study, the overall movement and contractibility of the hydra were reduced after coragen treatment. Hydra possess a primitive neuromuscular system. Recently, using calcium imaging technique, scientists have demonstrated activation of ectodermal and endodermal epitheliomuscular tissue that participate in dynamic kinetics of hydra (Szymanski and Yuste, 2019).
The reduced motility observed in coragen treated hydra might be due to effects of coragen on the epithelial muscular tissue. It would be interesting to investigate the mechanism further.

Higher doses of coragen led to immediate death and disintegration in $100 \%$ of the treated hydra whereas longer exposures to low concentration of coragen were necessary to exhibit the toxic effects in the present study. The lethal effects of chlorantraniliprole on non-target species like crayfish in rice-crayfish cropping system has been reported (Barbee $e t$ al., 2010). Coragen was found to hamper the regeneration also, which is in accordance with the earlier observations that pesticides and insecticides may affect crucial life processes of animals. The reproduction capability of worker bumblebees is shown to be suppressed by dietary intake of chlorantraniliprole, an active ingredient of coragen (Smagghe et al., 2013). Chlorantraniliprole exposure is shown to reduce overall growth and formation of cocoons in Bombyx mori through accumulation of reactive oxygen species (ROS), down-regulation of genes related to oxidative phosphorylation pathway and antioxidant defense system in midgut of the animal (Hu et al., 2019). Coccinella septempunctata is a commonly used insect predator for controlling of many arthropod pests in integrated pest management. A recent study has proved significant reduction in the hatchability of eggs, pupation rate, emergence of adults of Coccinella septempunctata due to treatment with chlorantraniliprole, eventually resulting in low population levels of the beneficial species (He et al., 2019). Similar study by another group on the multicolored Asian lady beetle Harmonia axyridis, a nonspecific predator, showed impaired population 
growth due to sublethal concentrations chlorantraniliprole (Nawaz et al., 2017). Chlorantraniliprole (in a range of 5 to 100 $\mathrm{ppm}$ ) is shown to be highly toxic to eastern subterranean termites resulting in $100 \%$ mortality within 14 days post-exposure (Buczkowski et al., 2012). Exposure to chlorantraniliprole for more than 14 days led to reduction in weight and increased ROS levels in earthworms (Liu et al., 2018). Chlorantraniliprole is shown to interfere with the vertebrate embryogenesis process also (Borgave et al., 2020).

The present study thus demonstrates toxic effects of coragen on non-target aquatic species. It further emphasize precocious use of coragen in agricultural fields as it interferes with biological phenomenon like regeneration and leads to lethal effects in a supposedly immortal organism like hydra. There are hints of elevation in ROS and impairment of antioxidant mechanism due to chlorantraniliprole (Liu et al., 2018; Hu et al., 2019). Impact of ROS in planarian regeneration has been demonstrated by Pirotte $\mathrm{Hu}$ et al. (2015). In light of these studies, it would be interesting to elucidate the mechanism by which coragen interferes with the regeneration process in hydra.

\section{Acknowledgement}

The author acknowledges Department of Science and Technology-FIST for the funding for DM 1000 microscope and Department of Biotechnology for the grants under 'Star college scheme' through which the Future WinJoe camera was purchased.

\section{References}

Barbee GC, McClain WR, Lanka SK and Stout MJ. (2010) Acute toxicity of chlorantraniliprole to non-target crayfish (Procambarus clarkii) associated with rice- crayfish cropping systems. Pest Manag Sci. 66: 996-1001.

Blaise C, Gagné F, Harwood M, Quinn B and Hanana H. (2018) Ecotoxicity responses of the freshwater cnidarian Hydra attenuata to 11 rare earth elements. Ecotoxicol Environ Saf. 163: 486-491.

Borgave S, Mhaske S, Gadekar M and Bhagde R. (2020) Toxicity of coragen on early zebrafish embryo. Intern J Res Anal Rev. 7: 251-263.

Bouchard R, Pattarini R and Geiger JD. (2003) Presence and functional significance of presynaptic ryanodine receptors. Prog Neurobiol. 69: 391-418.

Buczkowski G, Scherer CW and Bennetti GW. (2012) Toxicity and horizontal transfer of chlorantraniliprole in the eastern subterranean termite. J Econ Entomol. 105:1736-1745.

Dutta K, Ali M, Najam A, Kumar R and Kumar A. (2014) Ameliorative effect of seed extract of Pterocarpus santalinus on coragen induced haematological alterations and serum biochemical changes in Charles Foster rats. J Toxicol Environ Health Sci. 6:194-202.

He F, Sun S, Tan H, Sun X, Shang D, Yao C, Qin C, Ji S, Li $X$, Zhang J and Jiang X. (2019) Compatibility of chlorantraniliprole with the generalist predator Coccinella septempunctata L. (Coleoptera: Coccinellidae) based toxicity, life-cycle development and population parameters in laboratory microcosms. Chemosphere 225:182-190.

Hu J, Li M, Lu Z, Mao T, Chen J, Wang H, Qu J, Fang Y, Cheng X, Li J, Li F and Li B. (2019) The mechanism of damage in the midgut of Bombyx mori after chlorantraniliprole exposure. Ecotoxicol. 28: 903-912.

Karntanut W and Pascoe D. (2002) The toxicity of copper, cadmium and zinc to four different Hydra (Cnidaria: Hydrozoa). Chemosphere 47: 1059-1064.

Liu T, Wang X, Chen D, Li Y and Wang F. (2018) Growth, reproduction and biochemical toxicity of chlorantraniliprole in soil on earthworms (Eisenia fetida). Ecotoxicol Environ Saf. 150: 18-25.

McKinley K, McLellan I, Gagné F and Quinn B. (2019) The toxicity of potentially toxic elements $(\mathrm{Cu}, \mathrm{Fe}, \mathrm{Mn}$, $\mathrm{Zn}$ and $\mathrm{Ni}$ ) to the cnidarian Hydra attenuata at environmentally relevant concentrations. Sci Total Environ. 665: 848-854. 
Mishra A, Chandiraseharan V, Jose N and Sudarsanam T. (2016) Chlorantraniliprole: An unusual insecticide poisoning in humans. Indian J Crit Care Med. 20: 742-744.

Nawaz M, Cai W, Jing Z, Zhou X, Mabubu JI and Hua H. (2017) Toxicity and sublethal effects of chlorantraniliprole on the development and fecundity of a non-specific predator, the multicolored Asian lady beetle, Harmonia axyridis (Pallas) Chemosphere 178: 496-503.

Pirotte N, Stevens An-S, Fraguas S, Plusquin M, Roten AV, Belleghem FV, Paes en R, Ameloot M, Cebria F, Artois T and Smeets K. (2015) Reactive oxygen species in planarian regeneration: an upstream necessity for correct patterning and brain formation. Oxid Med Cell Longev. 2015: 1-20.

Quinn B, Gagné F and Blaise C. (2012) Hydra, a model system for environmental studies. Int J Dev Biol. 56: 613-625.

Riedl V, Agatz A, Benstead R and Ashauer R. (2018) A standardized tritrophic small-scale system
(TriCosm) for the assessment of stressor-induced effects on aquatic community dynamics. Environ Toxicol Chem.-37: 1051-1060.

Smagghe G, Deknopper J, Meeus I and Mommaerts V. (2013) Dietary chlorantraniliprole suppresses reproduction in worker bumblebees. Pest Manag Sci. 69: 787-791.

Solis M, Bonetto C, Marrochi N, Paracampo A and Mugni H. (2018) Aquatic macroinvertebrate assemblages are affected by insecticide applications on the Argentine pampas. Ecotoxicol Environ Saf. 148:11-16.

Staley Z, Harwood, V and Rohr J. (2015) A synthesis of the effects of pesticides on microbial persistence in aquatic ecosystems. Crit Rev Toxicol. 45): 813-836.

Szymanski JR and Yuste R. (2019) Mapping the wholebody muscle activity of Hydra vulgaris. Current Biol. 29: 1807-1817. 